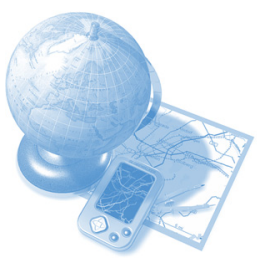

Derek Raine

Department of Physics and Astronomy

University of Leicester

Leicester LE1 7RH

jdr@le.ac.uk

\section{Project LeAP: Learning in Astronomy and Physics}

\begin{abstract}
The aim of the project was to raise awareness of problem-based learning (PBL) in physics through the development of exemplars relevant to the HE context in the UK, and through road shows and seminars on the background to PBL. There is also a LeAP web site devoted to Physics PBL. The project was carried out in collaboration with partners in Hertfordshire, Reading and Sheffield and the results published by the Physical Sciences Subject Centre as a practice guide to PBL in Physical Sciences. This article will discuss some of the evidence gathered by the project on PBL in physics worldwide, and report on some of the implementations that are beginning supported by the project as well as future developments in the Physics Innovations Centre for Excellence.
\end{abstract}

\section{Introduction}

Tradition is a limitless resource. It can be used endlessly to defend why everything is as it is and not otherwise. And indeed, if things have worked in the past why should they not work now? Tradition is particularly strong in a subject like physics: Newton's laws have not changed for three hundred years, Maxwell's for one hundred and fifty and Einstein's for a hundred (give or take, here and there). The current way we express these and other laws, and the way we understand these branches of physics, has been taught to generations of physics students - with obvious success, since physics has not died out (yet). So why change the way we teach?

Where to begin! The subject content may have stayed the same but the learning environment has changed. The traditional specification of the physics curriculum is manifestly uninspiring to the majority of students, who are led to believe that physics is a set of arbitrary lists of equations in areas such as heat, light, sound, gravity, ... that are disconnected with each other and with anything meaningful in 'real life'. A magnifying glass may well once have competed with a flower press for a child's attention, but it does not appear to enthrall the videogames generation.

It is also tradition that physics presents a long and arduous learning curve that has to be climbed by means of apparently endless end-of-chapter exercises, of no intrinsic interest, in order to get to the really interesting stuff. But this interesting stuff is located somewhere at the end of a rainbow for most students, who don't in fact ever get to [address] profound questions about the universe [or]... understand the complex physical and environmental systems in which we exist ${ }^{1}$.

It is a traditional claim that physics is a linear subject that requires mastery of the fundamentals before moving on. This would not be worth contesting were it not for the fact that curriculum designers and lecturers appear to use this fundamentalism to justify the boring bits. In fact, the linearity is much over-hyped. One is rarely building on firm foundations: the understanding of most students is partial and inaccurate and only deepens through repeated exposure in different contexts. And it seems to have been forgotten that 40 years ago at least one A-level physics syllabus contained no mechanics: it is indeed possible to start with light, or heat or... . Of course, this approach loses the logical structure of physics, but it is a mistake to try to impress that structure on students who lack experience beyond that of the early undergraduate years. In other words, to my mind the logical structure of physics only appears logical in hindsight.

And finally, as a discipline, we keep repeating the mantra that physics is interesting to anyone who cares to think about it. I'm not sure about this: I think what physicists find interesting is being physicists doing physics, not taking notes on what they are being told. 
Seen from many of our 'traditional' institutions the declining popularity of physics is a bit of an illusion. Measured purely in terms of numbers of undergraduates many Departments have apparently never been healthier. It is however a real problem for the UK as a whole. So we must DO something!

So what about making it fun! I have no evidence beyond introspection to back this up, but I think that is a really BIG mistake. My own view is that we need to render physics more serious. By this I mean that we illuminate the way in which the study of science, and of physics in particular, can offer a real opportunity to contribute to making a difference to the world. For, if physics is just a matter of individual gratification, why on earth is it important? I do not mean to equate 'serious' with 'dull' - physics can (should) be as much fun as you like, provided it is manifestly serious. An obvious example is medicine, the value of which is clear. It is this message that we need to substantiate.

How? Well my own favoured candidate is through problembased learning (PBL) and it is this that the FDTL4 project LeAP was supported to develop. It is certainly exciting to study outer space, but a student cannot do very much with the knowledge. I think Ohm's law should be even more exciting, because armed with an understanding of this, one can build a circuit to do things that no-one else in the history of the world has ever done before (if you will forgive a little bit of exaggeration to make the point). PBL poses a problem as a focus for student engagement, preferably a problem that is meaningful for students with their current experience, and exploits the benefits of collaborative learning by requiring students to work in groups. In this way it answers many of the difficulties of the traditional approach. It is student-centred, asking students to operate as physicists throughout, and starting from where they are with their own learning issues. It is capable of supporting multiple endpoints depending on student interests and abilities. It can underpin curricula of various shapes, by area of physics (if you insist) or by themes. And above all it shows that knowledge is important because of what you are able to do with it.

To develop these themes, in the course of project LeAP we ran various roadshows in Physics Departments across the country. We were able also to develop PBL in Leicester physics from a nascent concept through to a fully-fledged implementation across all years, although not across the board and not where other approaches are more efficient. We were also able to develop a new degree programme in Interdisciplinary Science taught wholly through $\mathrm{PBL}$, funded in part through FDTL4. Outside Leicester we were able to assist in the implementation of PBL in physics at Dublin City University, Dundalk Institute of Technology, The Universities of St Andrews, Bath, and Thames Valley as well as in Science Foundation Degrees in Newman College, Birmingham and Wyggyston QE College, Leicester. In addition our survey showed that up to half of the Departments of Physics claimed to have some PBL in their curricula. We also spread (and received) the message at various international meetings in Korea, Finland, Mexico, Peru and the US.

It was never part of the project to take PBL into schools, although in the US it is at this level that PBL in Physics has perhaps made most progress. It was interesting though to get the reaction of at least some physics teachers at Institute of Physics meetings, where it was claimed that any variations from teaching-to-the-test in schools would be impossible because of the focus on league tables!

One of the most important aspects of a project is a legacy. Project LeAP was unusual for an FDTL project in that it did not have as an objective the production of teaching materials in a form for others to use. What then is the legacy of project LeAP? Our annual International Summer Workshop on PBL, now in its fifth year, will be continuing, under the umbrella of the חCETL (the Physics Innovations Centre for Excellence in Teaching and Learning). There is a guide to PBL published by the Physical Sciences Subject Centre and available through their web site that offers practical guidance to anyone thinking of adopting PBL. This highlights the many approaches to PBL across the world, but also offers advice from experience of how things can go wrong. The LeAP web site itself ${ }^{2}$ hosts a bank of problems that will continue to grow in number. And, again continuing as part of the חCETL, we are happy to share our experiences with anyone who thinks they might benefit from them. One project is of course not going to change the nature of physics teaching, certainly not over three years, but it can contribute to a momentum for change that will ensure the healthy survival of the discipline and, perhaps, a new tradition.

I am grateful to Dr Sarah Symons who managed the project wonderfully well, sometimes despite me. We are both grateful to our colleagues for their contributions to project LeAP, particularly our project partners Jim Collett at Hertfordshire, John McDonald at Reading and Craig Buttar and David Mowbray at Sheffield, the many assistants too numerous to mention individually who toiled to develop PBL materials and the staff at Leicester who helped to implement them. We enjoyed the unstinting support of the Physical Sciences Subject Centre and the Institute of Physics.

Project LeAP is supported by the Fund for the Development of Teaching and Learning (FDTL4)

\section{References}

1. From Physics - Building a Flourishing Future (loP)

2. www.le.ac.uk/leap 